\title{
Article \\ A Review on Unit Commitment Algorithms for the Italian Electricity Market
}

\author{
Maria Carmen Falvo ${ }^{1}\left(\mathbb{D}\right.$, , Stefano Panella ${ }^{1, *(\mathbb{C})}$, Mauro Caprabianca ${ }^{2}$ and Federico Quaglia ${ }^{2}$ (I) \\ 1 DIAEE-Department of Astronautics, Energy and Electrical Engineering, University of Rome Sapienza, \\ Via Eudossiana 18, 00184 Rome, Italy; mariacarmen.falvo@uniroma1.it \\ 2 TERNA, Viale Egidio Galbani 70, 00056 Rome, Italy; mauro.caprabianca@terna.it (M.C.); \\ federico.quaglia@terna.it (F.Q.) \\ * Correspondence: stefano.panella@uniroma1.it
}

Citation: Falvo, M.C.; Panella, S.; Caprabianca, M.; Quaglia, F. A Review on Unit Commitment Algorithms for the Italian Electricity Market. Energies 2022, 15, 18. https://doi.org/10.3390/en15010018

Academic Editor: Sungyun Choi

Received: 22 November 2021 Accepted: 17 December 2021 Published: 21 December 2021

Publisher's Note: MDPI stays neutral with regard to jurisdictional claims in published maps and institutional affiliations.

Copyright: () 2021 by the authors Licensee MDPI, Basel, Switzerland. This article is an open access article distributed under the terms and conditions of the Creative Commons Attribution (CC BY) license (https:// creativecommons.org/licenses/by/ $4.0 /)$.

\begin{abstract}
This paper focuses on the state-of-the-art of unit commitment (UC) and economic dispatch (ED) algorithms suitable for the Italian electricity market. In view of the spread of renewable energy systems (RES), the desired UC algorithm should be able to properly consider the uncertainty affecting key input variables into the formulation of the problem, as well as the different capabilities of dispatched power plants to provide ancillary services (e.g., voltage regulation). The goal of this paper is to resume the developments in UC and ED algorithms which occurred in the last decades, having a particular focus on alternating current (AC) security constrained (SC) approaches and stochastic ones, highlighting the advantages and weakness of each technique. This review is useful for the Italian TSO (Terna) to investigate what is the best solution to formulate a new algorithm to be potentially adopted in the framework of the Italian Ancillary Service Market, striving for an explicit modelization of stochastic variables and voltage constraints. This review is also useful to all system operators (SOs), independently to the market environment in which they operate, because UC algorithms are widely adopted to ensure real-time security of power systems. In conclusion, an SC-UC algorithm which takes into account both stochastic variables and AC formulation does not exist.
\end{abstract}

Keywords: alternating current optimal power flow (ACOPF); unit commitment (UC); optimal power flow (OPF); mixed integer nonlinear programming (MINLP); direct current optimal power flow (DCOPF); security constrained unit commitment (SCUC); ancillary service market (ASM); transmission system operator (TSO)

\section{Introduction}

Balancing electricity supply and demand is starting to become a challenging activity for most of the SOs around the globe because of the strong penetration of intermittent RESs. Moreover, intermittent RESs are gradually widening the variability of operating conditions, enlarging the related forecast confidence intervals, as well even in the short and very-short predicting horizon. In addition, voltage and frequency control is also impacted: power plants that are traditionally able to regulate voltage and frequency (mainly thermal units) are less and less committed in the electricity markets, while intermittent RESs are currently unable or only partially unable to deliver such services to the power system.

During 2020, the COVID-19 pandemic significantly lowered power system demand, creating similar (even if not exactly the same) conditions that could be expected in the future resulting from a further deployment of distributed RES generation. S. Bigerna et al. [1] analyzed the correlation between market power and the effect of COVID-19. In fact, the interval time analyzed in [1] from March to June 2020 was characterized by a massive demand reduction which was supplied by a huge RES deployment This event caused two important effects: an average price reduction respect the same period in 2019 and an increase of line congestion. The same C. Graf et al. [2] studied how the electricity market changed during the lockdown period, with a large share of renewables. P. Nikolaidis 
and A. Poullikkas [3] suggest a hybrid approach for solving UC considering the use of electricity storage systems, in order to manage the presence of uncertain and volatile input parameters, caused by the RESs. However, RESs don't cause only problems about the network security and reliability: S. Bigerna et al. [4] investigate how the RESs influence the electricity spot market too.

All these elements amplify the importance of the UC process, making it a key activity for achieving a reliable and efficient power system operation: an ideal UC process should be able to cope with forecast uncertainties (e.g., its results should be compatible with a wide range of operating scenarios that could materialize), ensuring that voltage and frequency control can be properly performed in real-time (e.g., a sufficient number of regulating units should be committed), considering also locational constraints.

Terna, the Italian Transmission System Operator (TSO), is in charge of ensuring the security of the Italian power system by means of procuring ancillary services from market participants throughout the Italian ancillary service market ("Mercato per il Servizio di Dispacciamento", MSD). For clearing the planning stage of the MSD Terna executes a Security constrained unit commitment and economic dispatch algorithm, accepting increase/decrease offers submitted from market participants, in order to adapt their schedules (as resulting from the previous energy markets). For this reason, Terna would automatically cope with all the security constraints (including voltage ones) and the stochastic variables inside the UC and ED formulation.

This paper aims to summarize the current state of the art about the resolution of the security constrained unit commitment and economic dispatch (SCUCED) problems. The review included in the present work is useful for the Italian TSO (Terna) to investigate what is the best solution to formulate a new algorithm to be potentially adopted in the framework of the Italian ancillary service market, striving for an explicit modelization of stochastic variables and voltage constraints. This review is also useful to all system operators (SOs), independently to the market environment in which they operate, because UC algorithms are widely adopted to ensure real-time security of power systems.

The paper is organized in six main Sections. Section 2 includes a summary description of the Italian electricity market, with a special focus on the Italian Ancillary Service Market (ASM). Section 3 describes the UC algorithm, focusing on its formulation and scope, as well as highlighting the main challenges incurred when is applied to large scale power systems. Section 4 describes the mathematical problem of optimal power flow (OPF), at the basis of UC and ED, distinguishing the alternating current (AC) OPF formulation, the direct current (DC) OPF formulation and the OPF based on the convex relaxation. Section 5 deals with the state of the art on the SCUCED resolution techniques proposed in the literature, also including references to some relevant study cases. Finally, Section 6 includes the study cases that focus on the SCUCED with uncertainties. In Section 7, key conclusions are summarized.

\section{Italian Electricity Market}

The Italian electricity market is organized in a Spot Electricity Market (named "Mercato a Pronti dell'Energia") and a Forward Electricity Market.

The Spot Electricity Market is divided into:

- A day-ahead market (DAM), called in Italy "Mercato del Giorno Prima (MGP)", as a first stage of the spot energy market;

- An intra-day market (IDM), called in Italy "Mercato Infragiornaliero (MI)", as a second stage of the energy market;

- An ancillary services market (ASM), called in Italy "Mercato del Servizio di Dispacciamento (MSD)".

The MGP is part of the single DAM coupling: a single auction, solved in the morning of the day before the delivery, which covers most of the EU countries applying the central algorithm called "EUPHEMIA" [5,6], accepts supply and demand offers submitted by market participants, computes zonal clearing prices and defines cross-zonal schedules 
(implicit allocation of cross-zonal capacity). The clearing algorithm [7] aims at maximizing the social welfare, so the structure of this market is not discriminatory and the accepted supply bids are paid at the Zonal Clearing Price (ZCP), while the accepted demand bids are paid, in Italy, at the National Single Price "Prezzo Unico Nazionale (PUN)".

An IDM is also in place, allowing market participants to adapt their schedules, in order to meet the technical constraints of their power plants and/or to better fit with load and RES forecasts. Till September 2021, the intraday market was based on seven auctions (similar to the MGP, but held at national level) which take place in the afternoon of the day before the delivery and during the delivery day itself. In September 2021, Italy joined the IDM coupling framework, dismissing the previous intraday markets and adopting the new regional approach. The new design is based on a continuous trading approach, starting in the afternoon of the day before the delivery and closing one hour before the delivery. The continuous trading approach is supported by three auctions held at regional level, which allow identifying a proper value for cross-zonal capacity as well as providing coordinated price signals to all the participants.

The MGP and MI are managed by the Italian Nominated Electricity Market Operator (NEMO), named in Italian "Gestore dei Mercati Energetici" (GME).

The Italian ASM, "Mercato dei Servizi del Dispiacciamento" (MSD), is the place where Terna, the Italian TSO, procures necessary ancillary services for ensuring the security of the power system. The MSD is divided into:

- A planning stage, named "MSD ex-ante", where Terna accepts offers and bids to guarantee the security of the system (e.g., to solve congestions and to create adequate reserve margins). In this stage, a one-hour market time unit (MTU) is currently adopted.

- A real-time balancing market, named "Mercato del Bilanciamento" (MB) in Italy, where Terna accepts offers and bids in real-time for balancing the electrical system and for solving residual congestions. In this stage, a 15 min MTU is currently adopted.

Both MSD ex ante and MB markets operate to maintain the security and reliability of the Italian power system [8].

The main objective of MSD is to adapt generation and, if participating in this market, load schedules raising from the energy markets, in order to solve network congestions and any other security issue (managing voltage and dynamic constraints) and to procure the secondary and tertiary reserve. Primary reserve is a response to the frequency variation of every production unit connect with the electrical grid, which is automatically delivered by the regulation speed of the generation group. From the event of frequency variation up to $15 \mathrm{~s}$ must be intervened the $50 \%$ of the primary reserve and this action must be ended into $30 \mathrm{~s}$. The provision of the primary reserve is mandatory in Italy for all the generation units bigger than 10 MVA. For doing that, the TSO solves a SCUCED problem: congestions and voltage constraints are solved in MSD redispatching generation units (or switching them on/off). Reserves are procured adapting generation schedules in a way that enough capacity is available and can be activated in due time (according to the reserve products it has been allocated on this capacity).

The secondary reserve (which corresponds to the EU automatic Frequency Restoration Reserve, aFRR) is involved into $180 \mathrm{~s}$ from the frequency variation. It aims to restore the primary reserve and compensate the gap between the electrical requirement and the production (restoring the exchanges with other countries at the scheduled value). This service is an automatic function, performed by a centralized controller located in the online control system of the TSO. In the planning stage, the SCUCED algorithm adapts the schedules in order to have enough secondary reserve band to cover the aFRR demand.

The tertiary reserve is divided into three categories:

- Ready reserve: this product has a full activation time of $15 \mathrm{~min}$, requiring a ramp rate of at least $50 \mathrm{MW} / \mathrm{min}$. Its goal is to rebuild the secondary power reserve band and maintain the system balance in the event of rapid changes in demand; 
- Spinning reserve (which corresponds to the manual Frequency Restoration Reserve, $\mathrm{mFRR}$ ): this product has a full activation time of $15 \mathrm{~min}$. Its goal is to replace the secondary power reserve band and the tertiary reserve ready;

- Replacement Reserve (RR): this product has a full activation time of $120 \mathrm{~min}$. Its goal is to replace the spinning tertiary reserve when activated and to face potential deviations in demand and RES infeed.

The TSO activates the available resources in the framework of the real-time balancing market to maintain the balance between the injections and withdrawals, to solve the electrical network congestions and to restore the secondary reserve band. In real-time balancing, Terna activates the procured resources for the tertiary reserve.

Terna joined the European platform for activating RR balancing energy (TERRE) in January 2021. The balancing process has been consequently integrated with an additional step, where RR energy supply and demand in shared at regional level.

\section{Unit Commitment (UC) Algorithm}

A key goal in power system operation is to meet the electricity demand at minimum cost, using an optimal mix of different power plants, according to their market offers. In order to match this criterion, ensuring at the same time the security and stability of the electricity network, a SCUCED algorithm is typically applied as it is considered one of the best options [9].

The scope of the UC activity is to determine the operational status of the production units (on/off) during the different MTUs of the delivery time horizon simulated, considering upward and downward availability of the production unit and coping with all their technical constraints (e.g., minimum uptime, minimum downtime, run-up rates, run-down rates). For this reason, the UC problem is a Mixed Integer problem. The UC solution methodologies can be classified into four categories: dynamic programming, Mixed Integer Linear Program (MILP) approaches, decomposition approaches and metaheuristics approaches [10].

In addition to the technical constraints of the production units, the algorithm shall cope with the security constraints of the power system, in order to deliver proper UCED choices. This would require the integration of a security assessment into the UCED problem, by means of an OPF formulation, which would also integrate the effect of contingency analysis. The OPF problem, in its exact formulation, is a non-linear problem.

Hence, the SCUCED is a mixed integer non-linear and non-convex problem, very challenging to be solved for large scale power systems, even on a single scenario, when runtime constraints are strict (e.g., for market operation). Adding complexity to this problem, introducing also the stochastic modelization would make the problem formulation too complex to be solved in due time.

An extended description of the UC problem can be found in $[9,11]$. For the scopes of this paper, it can be simplified as in the following.

The objective function of the SCUCED is shown in (1):

$$
\min \sum_{i \in G}\left(\sum_{t \in T}\left(p r_{\text {sell }}^{i, t} \cdot q_{\text {sell }}^{i, t}+p r_{\text {buy }}^{i, t} \cdot q_{\text {buy }}^{i, t}\right)\right)
$$

having as key constraint the energy balance in each point in time (2):

$$
\sum_{i \in G}\left(u^{i, t} \cdot\left(q_{\text {ini }}^{i, t}+q_{\text {sell }}^{i, t}+q_{\text {buy }}^{i, t}\right)\right)=\text { Demand }^{i, t} \forall t \in T
$$

where $p r_{\text {sell }}^{i, t}$ is the sell price offered by generation unit $i$ to increase its output from the initial schedule $q_{i n i}^{i, t}, p r_{b u y}^{i, t}$ is the buy price offered by generation unit $i$ to decrease its output, $q_{\text {sell }}^{i, t}$ and $q_{b u y}^{i, t}$ are the accepted incremental/decremental quantity and $u^{i, t} \in[0,1]$ is the commitment variable of the Production Unit. 
Additional constraints are typically added to this basic formulation, such as:

- Power flow equations;

- Transmission flow constraints;

- $\quad$ System spinning and operating reserve requirements;

- Ramp rate limitations;

- Startup and shutdown characteristics of units;

- Any additional security constraints provided by the SOs as resulting from other studies (e.g., dynamic assessments).

Currently, the SCUCED is typically solved by SOs around the world decomposing the problem into two iterative steps [12]:

- Market clearing algorithm, where the SCUCED is treated as a Mixed Integer problem without including power flow equations, but adding linear constraints to reflect security constraints, e.g., maximum loading of critical network elements (and contingencies);

- Security assessment module: in which the power flow problem is solved independently for each MTU, considering the base case scenario ( $\mathrm{N}$ state), as well as all the relevant contingencies (N-1 assessment). This module identifies potential binding critical network elements and contingencies and computes the related power transfer distribution factors: these are used to formulate linear constraints into the market clearing algorithm, in order to determine a UC and ED solution able to cope with such congestions. Sometimes, a DC formulation of the power flow equations is adopted and an AC final check is performed.

Anyhow, in this approach, voltage and reactive power constraints are difficult to treat and typically they are modelled in a simplified way: Low voltage issues are typically reflected limiting the transmission capacity on a set of lines, while high voltage issues are modelled introducing UC constraints (e.g., a minimum number of units, among a given set of power plants, shall be online). This approach, even if effective, could be inefficient, since the complex interaction between active and reactive power, as well as the ability to solve voltage issues by means of other remedial actions (e.g., managing shunt reactors, synchronous compensators, HVDCs) is only partially represented.

In Section 4 a detailed review of the available strategies to solve the OPF problem in the framework of a SCUCED is presented. The goal is to identify potential improvements to the current approach deriving from the most recent developments in the field of non-linear optimization problems.

In addition to the above-mentioned complexities, when a power system is highly penetrated by intermittent RESs and, also, when the electricity demand is strongly dependent on weather conditions, it is also crucial to account for the uncertainties in the expected operational scenario, in terms of hourly demand and RES generation profile and its geographical distribution. Stochastic approaches have been applied to the UC problem to cope with these sources of uncertainties: the problem is solved considering several operating scenarios each characterized by a probability of occurrence [13-15]. Typically, the following variables are treated as stochastic in these approaches: Solar generation; wind generation; demand; thermal capacity of overhead transmission lines, when dynamic line rating strategies are in place.

It is worth mentioning that the introduction of a stochastic SCUCED approach would require SOs to develop predictive algorithms, able to deliver not only the expected value of each variable, but also their distribution function or, even better, to generate scenarios with an assigned probability, considering also autocorrelation and cross correlation between variables and across the space.

\section{Optimal Power Flow Problem and Methods}

Several TSOs adopt an OPF to ensure the security of the power system in real-time while minimizing costs for redispatching. 
The OPF is an optimization problem aimed at minimizing an objective function that includes the (re)dispatching costs, considering the following variables: the active power generation $P_{g}$, the reactive power generation $Q_{g}$, the nominal line to line voltage $V$ and the voltage phase angle $\vartheta$.

The problem can be summarized with the Formula (3) [16]

$$
\begin{gathered}
\min _{P_{g}, Q_{g}, V, \vartheta} f\left(P_{g}, Q_{g}, V, \vartheta\right) \\
\text { s.t. } g(x) \leq 0 \\
h(x)=0
\end{gathered}
$$

where the $h(x)$ and $g(x)$ are constraints respectively of equality and inequality. The equality constraints concern the balance of electrical powers like the (4) and (5):

$$
\begin{aligned}
& P_{i}=\sum_{j=1}^{n} 3 E_{i} E_{j} Y_{i j} \cos \left(\vartheta_{i}-\vartheta_{j}-\gamma_{i j}\right) \\
& Q_{i}=\sum_{j=1}^{n} 3 E_{i} E_{j} Y_{i j} \sin \left(\vartheta_{i}-\vartheta_{j}-\gamma_{i j}\right)
\end{aligned}
$$

while the inequality constraints are the equation from (6) to (11):

$$
\begin{gathered}
P_{i j}^{2}+Q_{i j}^{2}=S_{\text {max }, i j}^{2} \\
I_{i, R e}^{2}+I_{i, I m}^{2} \leq I_{\text {max }}^{2} \\
P_{g, \text { min }} \leq P_{g} \leq P_{g, \text { max }} \\
Q_{g, \text { min }} \leq Q_{g} \leq Q_{g, \text { max }} \\
V_{i, \text { min }} \leq V_{i} \leq V_{i, \text { max }} \\
\vartheta_{i j, \text { min }} \leq \vartheta_{i j} \leq \vartheta_{i j, \text { max }}
\end{gathered}
$$

The (6) and (7) are the thermal limit of the $i$-th in terms of power and thermal capacity, the (8) and (9) are the maximum and minimum limits of the active and reactive power generated, finally the inequalities (10) and (11) represent the upper and lower limits allowed for the voltage $\mathrm{V}$ at the $i$-th node and for the angle phase $\vartheta$ between the $i$-th and $j$-th node of the electrical network.

Since the constraints described from (6) to (11) are convex functions the computational burden is not compromised at all, the non-convexity of the OPF problem comes from the power flow Equations (4) and (5). Another important thing is that the standard OPF formulation does not consider the UC problem, so the OPF does not resolve automatically the discrete variables. In fact, the UC problem introduces in the OPF a non-linearity caused by its discrete variables, which increase the computational burden of the algorithm.

Because the OPF problem was first formulated in the 1960s, a multitude of solution algorithms have been proposed, increasing the speed of solution and minimizing the risk of non-convergence by the algorithm. This allows an industrial practical implementation in power systems management contexts.

Existing OPF algorithms can be divided in the following categories:

- OPF methods with the strict AC network model: in these methods, the original formulation of the power flow Equations (4) and (5) of the AC network model, are considered during the optimization. The relevant OPF model is also referred to as the ACOPF model. Nonlinear optimization techniques are used to solve the OPF problem, because of the nonconvexity and the non-linearity of the ACOPF $[17,18]$.

- OPF methods with linearized network models: the non-linear equations are replaced with a linearized and approximate one, this method is called "DC method". Thereby the computational burden is reduced, the computational efficiency can be dramatically 
improved, and convergence can be guaranteed, although the modelling accuracy is sacrificed. This formulation is widely used in the power sector and in market operation thanks to its ability to cope with strict market timings $[19,20]$.

- OPF methods based on convex relaxation: this approach relaxes the power flow Equations (4) and (5) into inequalities that define a convex region. These techniques approximate better the exact result of the problem than the DC form, but require an increased computational effort. The convex relaxation method is a compromise between the OPF AC model and the DC one [21,22].

Each of them presents vantages and disadvantages.

\subsection{OPF Methods with ACOPF}

The non-linearity and nonconvexity of the ACOPF are caused by the power flow Equations (4) and (5), which keep the original form without linearization. For OPF methods with the strict AC network model, general-purpose solution algorithms for nonlinear programming (NLP) problems can be applied, including the Newton method, linear programming (LP) methods and metaheuristics methods.

Among the NLP algorithms, the LP approach takes advantage of weak coupling between the active and reactive power and the quasilinear relationship between $P$ and $\vartheta$. For some LP approaches, the OPF optimization problem is decoupled into active and reactive subproblems [23].

Another method to solve ACOPF is an approximation with the Taylor series stopped at the first order [17], to linearize both the power flow equation and the voltage and current constraints, which have a quadratic dependence [24,25].

The approaches described above differ from the DC ones because they maintain the reactive power Equation (5), which instead is not considered in the DC approach as described in Section 4.2.

Actually, it is very difficult for SOs to apply these algorithms in clearing markets or DAMs or real-time scheduling, as long as their computational convergence cannot be guaranteed, although it has the advantage of introducing the voltage constraints into its formulation through exact modelling.

\subsection{OPF Methods with Linearized Network Models (DC Method)}

OPF methods with linearized network models intend to linearize the power flow Equations (4) and (5) and facilitate the linear formulation of the OPF model following a DC approach. OPF methods with linearized network models are preferred by system operators because of their desirable computational performance. Another advantage of the linear optimization model is its transparency. The influencing factors in the OPF model are linearly coupled.

The hypothesis of the DC models are the following:

- The active power Equation (4) is considered, but the reactive power Equation (5) is not;

- The network elements considered are only represented by their own longitudinal reactance;

- Neglecting these parameters, the nodal voltage module is considered constant and the $\Delta \vartheta$ between the $i$-th and $j$-th node are "close", i.e., $\vartheta_{j}-\vartheta_{i} \approx 0$.

The matrix of nodal admittances becomes a matrix of susceptances $[B]$ linked only to the longitudinal susceptances of the branch. The sine is approximated into the difference of its argument.

$$
P_{i}=\sum_{j=1}^{n} 3 E_{i} E_{j} Y_{i j} \cos \left(\vartheta_{i}-\vartheta_{j}-\gamma_{i j}\right)=\sum_{j=1}^{n} B_{i j} \sin \left(\vartheta_{i}-\vartheta_{j}\right) \approx \sum_{j=1}^{n} B_{i j}\left(\vartheta_{i}-\vartheta_{j}\right)
$$

The (12) can be written in the (13) form:

$$
[P]=[B][\Delta \vartheta] \Longrightarrow[\Delta \vartheta]=[B]^{-1}[P]
$$


Concluding, the DC approximation methods are widely used by system operators to solve the OPF problems in electrical markets, thanks to their low computational burden and the convergence can be guaranteed. In fact, the DCOPF is a convex problem and for this reason, it is easily solved. However, the DCOPF lacks the accuracy of the solution because the reactive power is not considered, so the voltages are set at their rated value. The drawback of DCOPF is that the voltage constraints are not considered in the algorithm, which are a prerogative for the new algorithm wanted by Terna.

\subsection{OPF Methods Based on Convex Relaxation}

Since the power flow equations are non-linear, these describe a non-convex region which makes the OPF hard to solve. The task of the convex relaxation consists of modifying the (4) and (5), in order to define a convex region.

An example is Z. Yang et al. [16] who express a model of OPF methods based on convex relaxation.

If the optimal solution of the relaxed model is a feasible solution to the original OPF problem, the relaxed model achieves the global optimum of the original OPF model. That means if the optimal solution of the relaxed model is feasible, then it is also the solution of the original problem. Since the UC-OPF is a nonconvex MINLP, in the past a popular method has been introduced called Lagrangian Relaxation (LR). It consists to solve a relaxed problem, but it suffers from the existence of a duality-gap and finds another problem to solve the original one.

There are several relaxation techniques in the literature, the most used are: Semidefinite Programming Relaxation (SDP), Second-Order Cone Programming Relaxation (SOCP) and Quadratic Programming Relaxation (QP).

\section{Review on OPF Methods for UC and ED}

This section includes some key study cases found in the literature, illustrating the application of the SCUCED resolution techniques. In order to verify the state of the art about OPF based UC and ED problems, an extensive literature analysis was conducted, including also relevant existing reviews [16,24,26].

In [27] A. Castillo et al. propose the application of a Generalize Bender Decomposition (GBD) method through successive iterations with the Taylor series approximation, dividing the MINLP problem into a master MILP problem and several NLP sub-problems. These last ones are solved by using a Successive Linear Programming approach. The GBD is a particular case of Outer Approximation (OA), which is applied both to classic UC and SCUC. The difference between the OA and the GBD is that the lower bounds of the last one are generally weaker, i.e., the lower bound predicted by the relaxed master problem of the $\mathrm{OA}$ is probably greater than or equal to that predicted by the master problem of the GBD.

The results of this proposed strategy are summarized in [27]: it show good performance, considering that the problem has been solved with a $2.2 \mathrm{GHz}$ Intel Core i7 and 16 GB RAM calculator. For the scope of this paper, it is very interesting to note that the UC based on the ACOPF is 5-10 times slower than a DCOPF. Moreover, the tests are performed on a power system 10 times smaller than the Italian one.

In [28], J. Liu et al. use an algorithm based on the multi-tree global optimization methodology, which iterates between a Mixed Integer lower-bounding problem and a nonlinear upper bounding problem. The results of this study show a performance comparison with the approach announced in [28], for the global case is used a 64-bit server with 24 CPUs (Intel(R) Xeon(R) CPU E5-2697 v2 @ 2.70 GHz) and 256 GB RAM. It is evident how increasing the size of the power system the optimality gap of the local approach increase too, while the optimality gap of the global approach remains substantially contained, nevertheless with calculation times significantly longer and completely incompatible for market operation. So, the time of solution is from $3.6 \mathrm{~s}$ for the 6-bus system to $115.23 \mathrm{~s}$ for IEEE-118 system, however even here the tests are performed on a power system 10 times smaller than the Italian one. 


\section{The Stochastic Algorithms Review}

When a power system is highly penetrated by intermittent RESs, and when the electricity demand is strongly dependent on weather conditions, it is crucial to account for the uncertainties in the expected operational scenario, in terms of hourly demand and RESs generation profile and its geographical distribution. Stochastic approaches have been applied to the UC problem to cope with these sources of uncertainties: the problem is solved considering several operating scenarios each characterized by a probability of occurrence [13-15].

Typically, the following variables are treated as stochastic in these approaches: solar generation, wind generation, demand, thermal capacity of overhead transmission lines (when dynamic line rating strategies are in place).

There are at least three solution techniques that have been proposed for managing uncertainties in SCUC, based on the Bender Decomposition, dividing the original problem into a master one and other sub-problems:

- Stochastic programming (SP): A renowned optimization technique to solve SCUC problem with uncertainties. In the SP approach, power system uncertainties are represented by a set of scenarios for the possible realization of different uncertainties. SP technique is based on a scenario tree in which uncertainty is supposed to be known in each node as long as uncertainty may be discretized on the tree, essentially the quantity for solving a deterministic large-scale UC problem. Every scenario is attributed a certain probability for its realization;

- Robust optimization (RO): $\mathrm{RO}$ is an alternative technique for dealing with uncertainties in the SCUC problem. RO uses the notion of uncertainty set be less demanding on the representation of uncertainty, which assembles the adverse events against which we wish to protect ourselves. This uncertainty set considers a limited level of information on uncertain quantities, namely the mean value and some estimate of the variance or a range of possible variations around the mean;

- Chance constrained optimization (CCO): The CCO is the third approach to handling the uncertainties in the hourly SCUC problem, in which temporal constraints can be violated with a predefined level of probability. CCO appears as a good alternative to select the tradeoff between cost and robustness of the electrical network.

I. Abdou and M. Tkiouat [24] compare the three solutions just described.

Stochastic algorithms define the stochastic variables, i.e., wind, solar, energy requirements and plants availability, which are linked with a probabilistic function called "Probability Distribution Functions". G.-C. Juan Sebastián et al. [29] solve the UC and ED problems with several scenarios simultaneously, in order to choose the solution which minimizes the expected cost over all the scenarios.

Another technique used in machine learning in order to manage the uncertainties is the Bayesian Optimization, which is one of the most and efficient approaches in terms of the number of function evaluations required [30].

P. Nikolaidis et al. [31] propose a novel approach for addressing the robust UC problem in the presence of identical generating units ( 20 generating units characterized by identical cost coefficients and start-up costs), allowing uncertainty-aware prediction using a Bayesian regression technique, called Gaussian Process. The methodology of [31] was compared with a Lagrange Relaxation technique: the results are that the Bayesian Optimization is more performing. C. Ning and F. You [32] propose a novel data-driven adaptive robust optimization framework for the UC problem integrating wind power into smart grids, using a Bayesian approach with a 6-bus and IEEE 118-bus system. So the Bayesian Optimization is successfully applied to solve expensive black-box problems in engineering and machine learning, ideal to manage model-fitting problems [33].

With the stochastic algorithms, acceptable levels of violation constraints probability are fixed, so the algorithm can identify the lowest cost solution of (re)dispatching, guaranteeing a violation constraints' probability less than the threshold fixed. 
H. Wu et al. in [34] underline how the fixed thresholds, such as the loss-of-load probability accepted (LOLP) and probability of transmission line overload accepted (TLOP), indicators can affect the rotating reserve costs. A mathematical formulation of LOLP is described in [15]. In particular, the rotating reserve costs decrease with the increase of the probability of losing the electrical load.

The LOLP and TLOLP indicators are used as constraints in [35], which presents a UC problem with uncertain loads and wind power, solved by a chance constrained two-stage stochastic programming formulation for the stochastic day-ahead scheduling.

The OPF based SCUC computational burden raises a lot considering the introduction of stochastic variables, with several operational scenarios. That means the computational timings raise too.

This is highlighted in [19], where a stochastic SCUC is described and tested on a model of the California power system with 225 bus, 375 lines and 130 generators. This paper presents a DC formulation and despite of the powerful hardware used, the computational timings of $5-15 \mathrm{~h}$ are not compatible with the Terna necessity. A. Papavasiliou et al. [19] show the several cases studied, considering about 1000 scenarios with N-1 security criteria, for each season of the year.

Papavasiliou and Oren [36] compare in their paper two approaches for committing locational reserves: stochastic unit commitment and a hybrid approach of scenario-based security-constrained commitment which studied parallel algorithms developed for solving the resulting models, based on Lagrangian relaxation and Benders decomposition. In [36] we can distinguish the different computational burdens of both the solution techniques proposed, by analyzing the electrical network system of the State of California, which is composed of 225 buses, 375 lines and 130 generators. The running time of the Lagrangian relaxation algorithm ranges between $15.8 \mathrm{~h}$ for the fully serial implementation to $47.7 \mathrm{~min}$ in the fully parallel implementation, is also underlined how the marginal benefits of parallelization vanish beyond 15 processors (such as the Benders decompositions solution). The advantage of the Bender decompositions solutions is evident, in fact the entire model requires $26.6 \mathrm{~min}$ to solve in a fully serial implementation, versus $14.8 \mathrm{~min}$ in a fully parallel implementation. The speedup of the Benders decompositions algorithm is due to the parallelization of the continuous DC.

Table 1 summarizes the mains technique for solving the ACOPF considering the UC, showing advantages and weaknesses.

Table 1. Comparison of SCUC technique with uncertainties.

\begin{tabular}{|c|c|c|}
\hline Method & Advantages & Weaknesses \\
\hline Lagrangian Relaxation & $\begin{array}{l}\text { It is able to process with non-linear constraints } \\
\text { only by relaxing them. } \\
\text { It can be decomposed in subproblems. }\end{array}$ & It suffers from the existence of a duality gap. \\
\hline Bender Decomposition & $\begin{array}{l}\text { It can be separated into independent problem } \\
\text { and sub-problem. }\end{array}$ & Low speed of convergence. \\
\hline $\begin{array}{l}\text { Mixed Integer Linear } \\
\text { Programming }\end{array}$ & $\begin{array}{l}\text { Is a powerful modelling tool, with a great ability } \\
\text { to react in a globally optima solution. }\end{array}$ & $\begin{array}{l}\text { It takes a long time compared to fast methods } \\
\text { like heuristics. } \\
\text { Is not good in treating largescale problems. }\end{array}$ \\
\hline Non-linear Programming & $\begin{array}{l}\text { Good modelization of power } \\
\text { generation characteristics. }\end{array}$ & $\begin{array}{l}\text { It enhances the problem dimension } \\
\text { and complexity. }\end{array}$ \\
\hline Hybrid Meta-Heuristic & $\begin{array}{l}\text { Is capable of handling indifferentiable cost } \\
\text { functions and constraints }\end{array}$ & Its finetuning is a negative point. \\
\hline Bayesian Optimizzation & $\begin{array}{l}\text { Very used to get an optimal solution } \\
\text { with uncertainties. } \\
\text { Useful to solve black-box problems and } \\
\text { model-fitting problems. }\end{array}$ & $\begin{array}{l}\text { It is really complicated to perform } \\
\text { a modelization. }\end{array}$ \\
\hline
\end{tabular}




\section{Conclusions}

This paper shows the current state of the art about the solutions techniques available to solve the SCUCED problem, considering stochastic variables, in order to manage the strong penetration of the RESs. The authors have compared the features and the limits of most of the algorithms used and proposed in the recent scientific literature, showing how the DCOPF formulation is faster than the ACOPF.

This review is useful for the Italian TSO (Terna) to investigate what is the best solution to formulate a new algorithm to be potentially adopted in the framework of the Italian ASM, striving for an explicit modelization of stochastic variables and voltage constraints.

Actually, it seems very challenging to solve the SCUCED problem by adopting an ACOPF formulation together with an explicit modelization of the uncertainties (e.g., stochastic approaches) for industrial practical applications, and there are no references that show an algorithm which takes into account an AC formulation with stochastic variables. Anyhow, there are promising solutions that allow for solving a SCUCED based on an ACOPF approach or using stochastic approaches. This suggests that TSOs should evaluate which of these features are more relevant for the power system they are operating, accepting simplifications on the other part. The non-linearity and non-convexity of the problem led to computation time that, still today, are too long for market operation.

Table 2 summarizes the main features on the techniques proposed in the relevant scientific papers analyzed in the framework of the literature review.

Table 2. Summary on the techniques proposed in the scientific papers analyzed for the literature review.

\begin{tabular}{|c|c|c|c|c|c|c|c|c|c|c|}
\hline \multirow[b]{2}{*}{ Ref. } & \multicolumn{2}{|c|}{ Power Flow } & \multicolumn{2}{|c|}{ Security } & \multirow[b]{2}{*}{ Stoch. } & \multirow{2}{*}{$\begin{array}{c}\text { Voltage } \\
\text { Constraints }\end{array}$} & \multirow{2}{*}{ UC } & \multirow{2}{*}{ ED } & \multirow{2}{*}{ Study Case } & \multirow{2}{*}{ Time } \\
\hline & $\mathrm{AC}$ & DC & $\mathbf{N}$ & N-1 & & & & & & \\
\hline [19] & $x$ & $\checkmark$ & $\checkmark$ & $\checkmark$ & $\checkmark$ & $\checkmark$ & $\checkmark$ & $\checkmark$ & 225 buses, 375 lines, 130 gen. & 5-15 h, 1000 scen. \\
\hline [29] & $\checkmark$ & $x$ & $\checkmark$ & $x$ & $\checkmark$ & $\checkmark$ & $x$ & $\checkmark$ & 14,118 buses & N.D. \\
\hline [34] & $x$ & $\checkmark$ & $\checkmark$ & $\checkmark$ & $\checkmark$ & $x$ & $\checkmark$ & $\checkmark$ & 31,118 buses & $41 \mathrm{~s}$ \\
\hline$[37,38]$ & $x$ & $\checkmark$ & $\checkmark$ & $\checkmark$ & $\checkmark$ & $x$ & $x$ & $x$ & 37,118 buses & N.D. \\
\hline [39] & $x$ & $\checkmark$ & $\checkmark$ & $\checkmark$ & $\checkmark$ & $x$ & $\checkmark$ & $x$ & 24 buses & 25 s, 12 scen. \\
\hline [40] & x & $\checkmark$ & $\checkmark$ & $\checkmark$ & $\checkmark$ & $x$ & $x$ & $x$ & $30,118,300$ buses & $159.78 \mathrm{~s}, 480$ contingencies \\
\hline [41] & $x$ & $x$ & $\checkmark$ & $\checkmark$ & $\checkmark$ & $x$ & $\checkmark$ & $\checkmark$ & 100 gen. & $188.8 \mathrm{~min}$ \\
\hline [42] & & $x$ & & & $x$ & & x & & $\begin{array}{c}1000 \text { buses, } 1100 \text { lines, } \\
180 \text { gen. }\end{array}$ & N.D. \\
\hline [43] & $\checkmark$ & $x$ & $\checkmark$ & $x$ & $x$ & $\checkmark$ & $\checkmark$ & $\checkmark$ & 6,118 buses & $0.13 \mathrm{~s}$ \\
\hline [44] & $\checkmark$ & $\checkmark$ & $\checkmark$ & $\checkmark$ & $\checkmark$ & $\checkmark$ & $x$ & $\checkmark$ & 39 buses & N.D. \\
\hline [3] & $\checkmark$ & $x$ & $\checkmark$ & $\checkmark$ & $x$ & $\checkmark$ & $\checkmark$ & $\checkmark$ & 6,118 buses & $513 \mathrm{~s}$ \\
\hline [36] & $x$ & $\checkmark$ & $\checkmark$ & $\checkmark$ & $\checkmark$ & $x$ & $\checkmark$ & $\checkmark$ & 225 buses, 375 lines, 130 gen & $26.6 \mathrm{~min}$ \\
\hline [25] & $\checkmark$ & $x$ & $\checkmark$ & $x$ & $x$ & $\checkmark$ & $x$ & $\checkmark$ & Polish grid, $14,57,118$ buses & $2.7 \mathrm{~s}$ \\
\hline [45] & $\checkmark$ & $\checkmark$ & $\checkmark$ & $\checkmark$ & $x$ & $\checkmark$ & $\checkmark$ & $\checkmark$ & 24 buses & $7.50 \mathrm{~s}, 120$ contingencies \\
\hline [27] & $\checkmark$ & $x$ & $\checkmark$ & $\checkmark$ & $x$ & $\checkmark$ & $\checkmark$ & $\checkmark$ & $6,79,118$ buses & $110.17 \mathrm{~s}$ \\
\hline [46] & x & $\checkmark$ & $\checkmark$ & $\checkmark$ & $x$ & $\checkmark$ & $\checkmark$ & $\checkmark$ & $\begin{array}{l}1168 \text { and } 4672 \text { buses, } 1352 \\
\text { and } 2704 \text { prod. unit }\end{array}$ & $2-150 \mathrm{~min}$ \\
\hline [28] & $\checkmark$ & $x$ & $\checkmark$ & $\checkmark$ & $x$ & $\checkmark$ & $\checkmark$ & $\checkmark$ & $6,24,79,96,118$ buses & $8.5-14,400 \mathrm{~s}$ \\
\hline
\end{tabular}

Author Contributions: Conceptualization, M.C.F. and F.Q.; methodology, S.P. and F.Q.; validation, M.C., M.C.F. and F.Q.; formal analysis, S.P.; investigation, S.P.; resources, S.P.; data curation, S.P. and F.Q.; writing —original draft preparation, S.P.; writing—review and editing, M.C., M.C.F. and F.Q.; supervision, M.C., M.C.F. and F.Q. All authors have read and agreed to the published version of the manuscript.

Funding: This research received no external funding.

Institutional Review Board Statement: Not applicable.

Informed Consent Statement: Not applicable.

Data Availability Statement: Not applicable.

Conflicts of Interest: The authors declare no conflict of interest. 


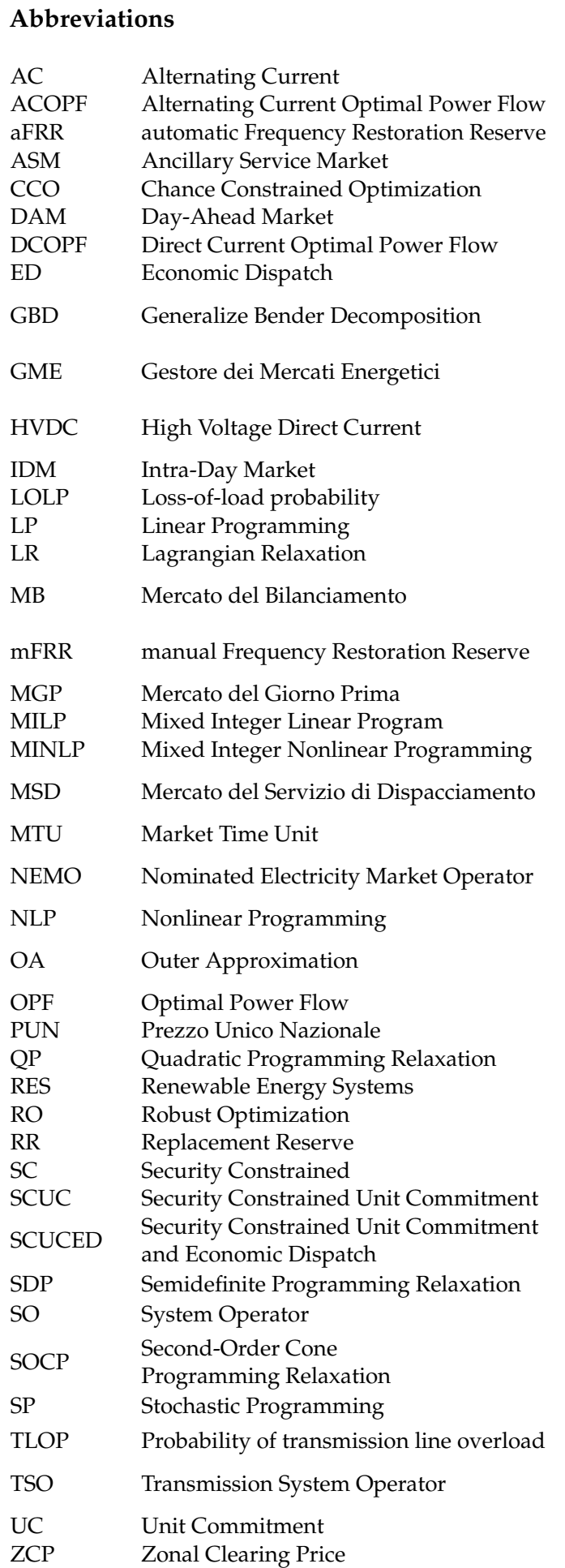

$P_{g} \quad$ Active power generated

$P_{i} \quad$ Active power from node $i$-th

$P_{i j} \quad$ Active power from node $i$-th to node $j$-th

$P_{g, \min } \quad$ Minimum level of active power generated

$P_{g, \max } \quad$ Maximum level of active power generated

$Q_{g} \quad$ Reactive power generated

$Q_{i} \quad$ Reactive power from node $i$-th

$Q_{i j} \quad$ Reactive power from node $i$-th to node $j$-th

Minimum level of Reactive

power generated

Maximum level of Reactive

power generated

Maximum apparent power from node $i$-th

$S_{\text {max }, i j} \quad$ to node $j$-th

$V \quad$ Nominal line-line voltage

$V_{i} \quad$ Nominal voltage line-line from $i$-th node

$V_{i, \min } \quad$ Minimum voltage line-line from $i$-th node

$V_{i, \max } \quad$ Maximum voltage line-line from $i$-th node

$E_{i} \quad$ Nominal phase-ground voltage from node $i$-th

$E_{j} \quad$ Nominal phase-ground voltage from node $j$-th

$\vartheta \quad$ Voltage phase angle

$\vartheta_{i} \quad$ Voltage phase angle from node $i$-th

$\vartheta_{j} \quad$ Voltage phase angle from node $j$-th

$\vartheta_{i j} \quad$ Voltage phase angle from node $i$-th to node $j$-th

$Y_{i j} \quad$ Ammetence from node $i$-th to node $j$-th

Ammetence phase angle from node $i$-th to node $j$-th

$I_{i, R e} \quad$ Real part of the current from node $i$-th

Imaginary part of the current from

node $i$-th

$I_{\max } \quad$ Maximum level of current

$B \quad$ Susceptances

$[P] \quad$ Matrix of active power

$[\Delta \vartheta] \quad$ Matrix of voltage phase angle variation

$[B] \quad$ Matrix of susceptances

$g(x) \quad$ Function of constraints inequality

$h(x) \quad$ Function of constraints equality

$i \in G \quad$ Interval of the $i$-th generation unit

$t \in T \quad$ Interval od the $t$-th hour of the day

$p r_{\text {sell }}^{i, t} \quad$ Sell price offered by generation unit $i$

$p r_{b u y}^{i, t} \quad$ Buy price offered by generation unit $i$

$q_{\text {sell }}^{i, t} \quad$ Accepted incremental quantity

$q_{b u y}^{i, t} \quad$ Accepted decremental quantity

$q_{i n i}^{i, t} \quad$ Initial quantity scheduled

$u^{i, t} \quad$ Commitment variable of the

Production Unit

\section{References}

1. Bigerna, S.; Bollino, C.A.; D'Errico, M.C.; Polinori, P. COVID-19 lockdown and market power in the Italian electricity market. Energy Policy 2021, 112700. [CrossRef]

2. Graf, C.; Quaglia, F.; Wolak, F.A. (Machine) learning from the COVID-19 lockdown about electricity market performance with a large share of renewables. J. Environ. Econ. Manag. 2021, 105, 102398. [CrossRef]

3. Nikolaidis, P.; Poullikkas, A. A novel cluster-based spinning reserve dynamic model for wind and PV power reinforcement. Energy 2021, 2021, 121270. [CrossRef]

4. Bigerna, S.; Bollino, C.A.; Ciferri, D.; Polinori, P. Renewables diffusion and contagion effect in Italian regional electricity markets: Assessment and policy implications. Renew. Sustain. Energy Rev. 2017, 68, 199-211. [CrossRef] 
5. Koltsaklis, N.; Dagoumas, A. Policy Implications of Power Exchanges on Operational Scheduling: Evaluating EUPHEMIA's Market Products in Case of Greece. Energies 2018, 11, 2715. [CrossRef]

6. Koltsaklis, N.E.; Zenginis, I.; Dagoumas, A.S. Assessing new block order options of the EUPHEMIA algorithm: An optimization model for the economic dispatch problem in power exchanges. Energy Rep. 2020, 6, 3119-3140. [CrossRef]

7. NEMO Committee. EUPHEMIA Public Description-Single Price Coupling Algorithm; NEMO Committee: Bruxelles, Belgium, 2019.

8. Caprabianca, M.; Falvo, M.C.; Papi, L.; Promutico, L.; Rossetti, V.; Quaglia, F. Replacement Reserve for the Italian Power System and Electricity Market. Energies 2020, 13, 2916. [CrossRef]

9. Padhy, N.P. Unit Commitment-A Bibliographical Survey. IEEE Trans. Power Syst. 2004, 12, 1196-1205. [CrossRef]

10. van Ackooij, W.; Lopez, I.D.; Frangioni, A.; Lacalandra, F.; Tahanan, M. Large-scale unit commitment under uncertainty: An updated literature survey. Ann. Oper. Res. 2018, 271, 11-85. [CrossRef]

11. Bai, X.; Wei, H. Semi-definite programming-based method for security-constrained unit commitment with operational and optimal power flow constraints. IET Gener. Transm. Distrib. 2009, 3, 182-187. [CrossRef]

12. CAISO. BPM for Managing Full Network Model. 2019. Available online: www.caiso.com (accessed on 13 December 2021).

13. Zheng, Q.P.; Wang, J.; Liu, A.L. Stochastic Optimization for Unit Commitment-A Review. IEEE Trans. Power Syst. 2015, 30, 1913-1924. [CrossRef]

14. Shiina, T.; Birge, J.R. Stochastic unit commitment problem. Int. Trans. Oper. Res. 2004, 11, 19-32. [CrossRef]

15. Wang, Q.; Wang, J.; Guan, Y. Stochastic Unit Commitment with Uncertain Demand Response. IEEE Trans. Power Syst. 2012, 28, 562-563. [CrossRef]

16. Yang, Z.; Zhong, H.; Xia, Q.; Kang, C. Fundamental Review of the OPF Problem: Challenges, Solutions, and State-of-the-Art Algorithms. J. Energy Eng. 2018, 144, 04017075. [CrossRef]

17. Castillo, A.; Lipka, P.; Watson, J.-P.; Oren, S.S.; O'Neill, R.P. A Successive Linear Programming Approach to Solving the IV-ACOPF. IEEE Trans. Power Syst. 2015, 31, 2752-2763. [CrossRef]

18. Ma, H.; Shahidehpour, S.M. Unit commitment with transmission security and voltage constraints. IEEE Trans. Power Syst. 1999, 14, 757-764. [CrossRef]

19. Papavasiliou, A.; Oren, S.S.; Rountree, B. Applying High Performance Computing to Transmission-Constrained Stochastic Unit Commitment for Renewable Energy Integration. IEEE Trans. Power Syst. 2015, 30, 1109-1120. [CrossRef]

20. Bienstock, D.; Shukla, A. Variance-Aware Optimal Power Flow: Addressing the Tradeoff Between Cost, Security, and Variability. IEEE Trans. Control Netw. Syst. 2019, 6, 1185-1196. [CrossRef]

21. Murillo-Sanchez, C.; Thomas, R.J. Thermal unit commitment with nonlinear power flow constraints. In Proceedings of the IEEE Power Engineering Society, 1999 Winter Meeting, New York, NY, USA, 31 January-4 February 1999; pp. 484-489.

22. Pareek, P.; Nguyen, H.D. State-Aware Stochastic Optimal Power Flow. Sustainability 2021, 13, 7577. [CrossRef]

23. Yang, Z.; Zhong, H.; Xia, Q.; Bose, A.; Kang, C. Optimal power flow based on successive linear approximation of power flow equations. IET Gener. Transm. Distrib. 2016, 10, 3654-3662. [CrossRef]

24. Abdou, I.; Tkiouat, M. Unit Commitment Problem in Electrical Power System: A Literature Review. Int. J. Electr. Comput. Eng. (IJECE) 2018, 8, 1357-1372. [CrossRef]

25. Yang, Z.; Bose, A.; Xia, Q. Optimal Power Flow in AC-DC Grids with Discrete Control Devices. IEEE Trans. Power Syst. 2017, 33 , 1461-1472. [CrossRef]

26. Håberg, M. Fundamentals and recent developments in stochastic unit commitment. Int. J. Electr. Power Energy Syst. 2019, 109, 38-48. [CrossRef]

27. Castillo, A.; Laird, C.; Silva-Monroy, C.A.; Watson, J.P.; O'Neill, R.P. The unit commitment problem with AC optimal power flow constraints. IEEE Trans. Power Syst. 2016, 31, 4853-4866. [CrossRef]

28. Liu, J.; Laird, C.D.; Scott, J.K.; Watson, J.P.; Castillo, A. Global solution strategies for the network-constrained unit commitment problem with AC transmission constraints. IEEE Trans. Power Syst. 2018, 34, 1139-1150. [CrossRef]

29. Sebastián, G.C.; Alexander, C.L.; Mauricio, G.E. Stochastic AC optimal power flow considering the probabilistic behavior of the wind, loads and line parameters. Ing. Investig. Tecnol. 2014, 15, 529-538. [CrossRef]

30. Brochu, E.; Cora, V.M.; De Freitas, N. A tutorial on Bayesian optimization of expensive cost functions, with application to active user modeling and hierarchical reinforcement learning. arXiv 2010, arXiv:1012.2599.

31. Nikolaidis, P.; Antoniades, A.; Chatzis, S. A Bayesian Optimization Approach for The Robust Unit Commitment of Identical Generating Units. In Proceedings of the 12th Mediterranean Conference on Power Generation, Transmission, Distribution and Energy Conversion (MEDPOWER 2020), Paphos, Cyprus, 9-12 November 2020; pp. 264-269.

32. Ning, C.; You, F. Data-Driven Adaptive Robust Unit Commitment Under Wind Power Uncertainty: A Bayesian Nonparametric Approach. IEEE Trans. Power Syst. 2019, 34, 2409-2418. [CrossRef]

33. Acerbi, L.; Ma, W.J. Practical Bayesian optimization for model fitting with Bayesian adaptive direct search. In Proceedings of the 31st Conference on Neural Information Processing Systems, Long Beach, CA, USA, 4-9 December 2017.

34. Wu, H.; Shahidehpour, M.; Li, Z.; Tian, W. Chance-Constrained Day-Ahead Scheduling in Stochastic Power System Operation. IEEE Trans. Power Syst. 2014, 29, 1583-1591. [CrossRef]

35. Wu, Z.; Zeng, P.; Zhang, X.P.; Zhou, Q. A Solution to the Chance-Constrained Two-Stage Stochastic Program for Unit Commitment with Wind Energy Integration. IEEE Trans. Power Syst. 2016, 31, 4185-4196. [CrossRef] 
36. Papavasiliou, A.; Oren, S.S. A comparative study of stochastic unit commitment and security-constrained unit commitment using high performance computing. In Proceedings of the 2013 European Control Conference (ECC), Zurich, Switzerland, 17-19 July 2013.

37. Guo, Y.; Baker, K.; Dall'Anese, E.; Hu, Z.; Summers, T.H. Data-Based Distributionally Robust Stochastic Optimal Power Flow_Part I: Methodologies. IEEE Trans. Power Syst. 2019, 34, 1483-1492. [CrossRef]

38. Guo, Y.; Baker, K.; Dall'Anese, E.; Hu, Z.; Summers, T.H. Data-Based Distributionally Robust Stochastic Optimal Power Flow-Part II: Case Studies. IEEE Trans. Power Syst. 2019, 34, 1493-1503. [CrossRef]

39. Sharifzadeh, H.; Amjady, N.; Zareipour, H. Multi-period stochastic security-constrained OPF considering the uncertainty sources of wind power, load demand and equipment unavailability. Electr. Power Syst. Res. 2017, 146, 33-42. [CrossRef]

40. Kimball, L.M.; Clements, K.A.; Davis, P.W. Stochastic OPF via Bender's method. In Proceedings of the 2001 IEEE Porto Power Tech Proceedings (Cat. No.01EX502), Porto, Portugal, 10-13 September 2001; Volume 3.

41. Ozturk, U.A.; Mazumdar, M.; Norman, B.A. A solution to the stochastic unit commitment problem using chance constrained programming. IEEE Trans. Power Syst. 2004, 19, 1589-1598. [CrossRef]

42. Berizzi, A.; Bovo, C.; Merlo, M.; Callegari, G.; Porcellini, M.; Pozzi, M. Second order sensitivities for constrained reactive optimal power flow. In Proceedings of the 2008 43rd International Universities Power Engineering Conference, Padua, Italy, 1-4 September 2008; pp. 1-7.

43. Chen, Y.; Xiang, J.; Li, Y. SOCP Relaxations of Optimal Power Flow Problem Considering Current Margins in Radial Networks. Energies 2018, 11, 3164. [CrossRef]

44. Rabiee, A.; Nikkhah, S.; Soroudi, A.; Hooshmand, E. Information gap decision theory for voltage stability constrained OPF considering the uncertainty of multiple wind farms. IET Renew. Power Gener. 2017, 11, 585-592. [CrossRef]

45. Amjady, N.; Dehghan, S.; Attarha, A.; Conejo, A.J. Adaptive Robust Network-Constrained AC Unit Commitment. IEEE Trans. Power Syst. 2016, 32, 672-683. [CrossRef]

46. Fu, Y.; Shahidehpour, M. Fast SCUC for Large-Scale Power Systems. IEEE Trans. Power Syst. 2007, 22, 2144-2151. [CrossRef] 